\title{
The relation between cognitive impairment severity and postural stability in the elderly
}

\author{
DOI: https://doi.org/10.5114/pq.2019.85150
}

\author{
Błażej Cieślik1, Dagmara Chamela-Bilińska², Bożena Ostrowska², Joanna Szczepańska-Gieracha² \\ ${ }^{1}$ Faculty of Pedagogy, Jan Dlugosz University, Częstochowa, Poland \\ 2 Department of Physiotherapy, University School of Physical Education, Wrocław, Poland
}

\section{Abstract}

Introduction. The purpose of this study was to evaluate the relationship between the severity of cognitive impairment and the results of stabilographic tests in elderly patients with cognitive impairment.

Methods. The study involved 42 participants aged 62-84 (mean $73.4 \pm 6.7$ ) years. The assessment of cognitive status was carried out with the Mini-Mental State Examination (MMSE). Included were subjects who obtained a score below 27, which is the cut-off score between the absence and presence of cognitive impairment. Postural stability was evaluated by using a force platform which registered the shifts of the centre of pressure (CoP) in the sagittal and frontal plane during two 32-second trials of quiet stance on a hard surface, with eyes open and with eyes closed. The CoP sway parameters were analysed.

Results. In the eyes-open test, we obtained a weak negative correlation of the MMSE and the mean radius of the CoP sway $(r=-0.37 ; p<0.05)$. Considering the values achieved in each plane, a statistically significant correlation was observed in all the parameters analysed in the sagittal plane. The eyes-closed test revealed a clear negative correlation between the MMSE results and all the studied parameters.

Conclusions. A significant relationship between the severity of cognitive impairment and the results of stabilographic parameters were noted. A negative correlation between the stabilographic parameters and the MMSE scores showed a linear deterioration of postural stability which progresses along with the decrease in cognitive functions.

Key words: dementia, centre of pressure, postural stability, balance, cognitive impairment

\section{Introduction}

Cognitive functions include a number of intellectual processes, such as short-term and long-term memory, linguistic processes (reading, writing, speech), visuospatial functions, constructive and executory functions, abstract thinking, as well as the processes of observation and perception of stimuli in the external environment. If running properly, the cognitive processes enable understanding, memorizing, and retrieving information, analysing situations and drawing conclusions, planning and decision making, maintaining attention despite distractions. Therefore, cognitive processes ensure an effective functioning of a person in the surrounding environment $[1,2]$.

Ageing of the body is related to the so-called age-associated memory impairment (AAMI) or, according to other authors, age-related cognitive decline (ARCD). Between normal ageing and mild dementia fall conditions termed as mild cognitive impairment $(\mathrm{MCl})$. Neither $\mathrm{MCl}$ nor the AAMI or ARCD mentioned above result in an impairment of the daily-life functions. If an impairment of cognitive function results in deterioration of daily living, dementia is recognized. According to the ICD-10 classification, dementias can be divided into three basic groups. Depending on the cause of the disorder, we identify vascular dementia, degenerative dementia (e.g. Alzheimer's disease, Pick's disease, dementia with Lewy bodies), or mixed dementia, where the two aetiologies coexist [3]. $\mathrm{MCl}$ is estimated to occur in $5-36 \%$ of the elderly population [4]. Moreover, the risk of dementia development in this group is significantly higher than in the general population [5]. Also, it is assessed that various types of dementia syndromes may affect $17.5 \%$ of the elderly population aged 60 years and above [6].

$\mathrm{MCl}$ has been classified as one of the fall risk factors in older people [7]. According to estimates, more than half of the older population regularly experience falls [8, 9]. However, the cause of the increased fall risk in this population is not entirely clear [10]. Agrawal et al. [11] have demonstrated that spatial cognitive skills deteriorate with age, which leads to the impairment of the vestibular system. Moreover, the decisionmaking processes are also disturbed; thus, older people with cognitive impairment may be prone to selecting inappropriate strategies for maintaining balance [12].

It seems that the increased risk of falls in this population is a very complex problem. On the one hand, it may result from the malfunctioning of the receptors involved in the process of maintaining balance. On the other hand, the phenomenon may be linked to the incorrect thinking processes which result in making wrong decisions when at risk of falling. Therefore, a further analysis of the causes of an increased fall risk in this population is very important and may help in developing more effective prevention programs. For this reason, we decided to evaluate the relationship between the severity of cognitive impairment and the results of stabilographic tests in elderly patients with cognitive impairment.

Correspondence address: Błażej Cieślik, Institute of Physical Education, Tourism and Physiotherapy, Faculty of Pedagogy, Jan Dlugosz University, ul. Waszyngtona 4/8, 42-200 Częstochowa, Poland, e-mail: blaze.cieslik@gmail.com 


\section{Subjects and methods}

\section{Participants characteristics}

The study involved 42 participants ( 27 women and 15 men) aged 62-84 (mean: $73.4 \pm 6.7$ ) years. The assessment of cognitive status was carried out with the Folstein's MiniMental State Examination (MMSE) [13, 14]. Included were subjects who obtained a score below 27 , which is the cutoff score between the absence and presence of cognitive impairment. This was a screening test, where the clinical cognitive impairment diagnosis was not yet determined. The mean MMSE score in the studied group was $19.11 \pm 6.23$ (range: 9-26). The exclusion criteria were the following: inability of unassisted movement or presence of mobility impairments preventing the performance of functional tests, memory impairment secondary to other degenerative problems like Parkinson's disease, stroke, severe cardiovascular diseases as vertigo or dizziness, amaurosis, major surgery or lower extremity fractures, and medication affecting balance. Table 1 presents the general characteristics of the studied population.

\section{Outcome measures}

Postural stability was evaluated by using a force platform which registered the shifts of the centre of pressure (CoP) in the sagittal and frontal plane during two 32-second trials of quiet stance on a hard surface, with eyes open and with eyes closed. The parameters of the CoP sway were analysed both without division into planes and with a differentiation between the sagittal plane and the frontal plane. The following variables were analysed: mean radius $(\mathrm{mm})$, sway area $\left(\mathrm{mm}^{2}\right)$, path length $(\mathrm{mm})$, mean velocity $(\mathrm{mm} / \mathrm{s})$, and number of sways. The sampling frequency was $20 \mathrm{~Hz}$.

\section{Statistical analysis}

Statistical analysis was performed with the Statistica 12 software. The results were presented by using descriptive statistics: the mean and the standard deviation. Given the distribution of data similar to normal, the statistical significance of the MMSE results correlation with the stabilographic parameters was checked with the Pearson's R test. The $t$-Student test for independent samples served to compare results between genders. The statistical significance was set at the value of $\alpha<0.05$.

\section{Ethical approval}

The research related to human use has complied with all the relevant national regulations and institutional policies, has followed the tenets of the Declaration of Helsinki, and has been approved by the Bioethics Committee of the Wroclaw Medical University.

\section{Informed consent}

Informed consent has been obtained from all individuals included in this study.

\section{Results}

In the eyes-open quiet stance trial, there was a significant negative correlation between the MMSE results and four investigated stabilographic parameters. Without specification of the planes, we obtained a weak negative correlation of the MMSE and the mean radius of the CoP sway ( $r=$ $-0.37 ; p<0.05$ ). Considering the values obtained in each plane, a statistically significant correlation was observed in

Table 2. Stabilographic parameters and MMSE score correlation, eyes-open test

\begin{tabular}{|l|c|c|c|}
\hline Parameter & Plane & MMSE & $p$ \\
\hline CoP mean radius & Total & -0.37 & 0.04 \\
\hline CoP sway area & Total & -0.34 & 0.06 \\
\hline \multirow{3}{*}{ CoP path length } & Total & -0.25 & 0.19 \\
\cline { 2 - 4 } & Sagittal & -0.49 & 0.00 \\
\cline { 2 - 4 } & Frontal & -0.19 & 0.33 \\
\hline \multirow{3}{*}{ CoP mean velocity } & Total & -0.28 & 0.28 \\
\cline { 2 - 4 } & Sagittal & -0.41 & 0.03 \\
\cline { 2 - 4 } & Frontal & -0.17 & 0.37 \\
\hline \multirow{2}{*}{ Number of sways } & Sagittal & -0.35 & 0.04 \\
\cline { 2 - 4 } & Frontal & -0.33 & 0.07 \\
\hline
\end{tabular}

MMSE - Mini-Mental State Examination, CoP - centre of pressure

Table 3. Stabilographic parameters and MMSE score correlation, eyes-closed test

\begin{tabular}{|l|c|c|c|}
\hline Parameter & Plane & MMSE & $p$ \\
\hline CoP mean radius & Total & -0.57 & 0.00 \\
\hline CoP sway area & Total & -0.65 & 0.00 \\
\hline \multirow{3}{*}{ CoP path length } & Total & -0.68 & 0.00 \\
\cline { 2 - 4 } & Sagittal & -0.60 & 0.00 \\
\cline { 2 - 4 } & Frontal & -0.69 & 0.00 \\
\hline \multirow{3}{*}{ CoP mean velocity } & Total & -0.67 & 0.00 \\
\cline { 2 - 4 } & Sagittal & -0.60 & 0.00 \\
\cline { 2 - 4 } & Frontal & -0.68 & 0.00 \\
\hline \multirow{2}{*}{ Number of sways } & Sagittal & -0.44 & 0.01 \\
\cline { 2 - 4 } & Frontal & -0.69 & 0.00 \\
\hline
\end{tabular}

MMSE - Mini-Mental State Examination, CoP - centre of pressure

Table 1. Participants' baseline characteristics (mean \pm standard deviation)

\begin{tabular}{|l|c|c|c|c|}
\cline { 2 - 5 } \multicolumn{1}{c|}{} & Total $(n=42)$ & Females $(n=27)$ & Males $(n=15)$ & $p$ \\
\hline Age $($ years $)$ & $73.43 \pm 6.77$ & $72.71 \pm 5.78$ & $74.70 \pm 8.35$ & 0.46 \\
\hline Body mass $(\mathrm{kg})$ & $66.21 \pm 12.91$ & $60.58 \pm 10.55$ & $76.61 \pm 10.13$ & 0.00 \\
\hline Height $(\mathrm{cm})$ & $162.70 \pm 9.18$ & $157.03 \pm 4.10$ & $173.03 \pm 5.90$ & 0.00 \\
\hline BMI $\left(\mathrm{kg} / \mathrm{cm}^{2}\right)$ & $24.93 \pm 3.90$ & $24.54 \pm 4.14$ & $25.62 \pm 3.53$ & 0.78 \\
\hline MMSE & $19.11 \pm 6.23$ & $20.27 \pm 6.52$ & $17.02 \pm 5.49$ & 0.18 \\
\hline
\end{tabular}


all the parameters analysed in the sagittal plane. The MMSE result correlated significantly with the CoP path length $(r=$ $-0.49 ; p<0.01)$, CoP mean velocity $(r=-0.41 ; p<0.05)$, and the number of sways $(r=-0.35 ; p<0.05)$. In the frontal plane, no statistically significant correlations were detected. Detailed data are presented in Table 2.

Increasing the difficulty of the trial by instructing the subject to close their eyes revealed a clear negative correlation between the MMSE results and all the studied parameters. The number of sways correlated at a moderate level $(r=-0.44$; $p<0.05)$, while the remaining results demonstrated a strong correlation with the MMSE (Table 3).

\section{Discussion}

The purpose of the study was to identify the relationship between the severity of cognitive impairment and the results of stabilographic tests in older patients with cognitive impairment. When analysing the correlation results, we may observe that in both trials the correlation is negative, i.e. the higher the MMSE score, the lower the postural stability parameters and vice versa. In other words, the better the cognitive function of a person, the better their balance control.

In the eyes-open postural stability trial, we may notice a statistically significant correlation between the MMSE scores and the stabilographic parameters in the sagittal plane. Both the CoP path length and CoP mean velocity, as well as the number of sways proved to correlate moderately with the severity of cognitive impairment. The above findings suggest that the process of maintaining balance becomes more chaotic with the progressing deterioration of cognitive function. During the quiet stance in patients with greater cognitive impairment, the CoP travels faster, farther, and more frequently in the sagittal plane. The result coincides with studies comparing postural stability in elderly healthy subjects and elderly subjects with cognitive impairment $[15,16]$.

In the anteroposterior balance control, a major role is played by muscles of the lower extremity, mainly the quadriceps femoris and the triceps surae muscles [17]. Among older people, the strength of the quadriceps femoris muscle significantly affects the correct balance control and when weakened, it significantly lowers the postural stability in this group [18, 19]. In our previous studies, we demonstrated a significant correlation between the severity of cognitive impairment and the strength of the quadriceps femoris muscle [20]. By analysing the above findings, one may conclude that lower values of stabilographic parameters in the sagittal plane may be linked to the limited strength of the quadriceps femoris muscle, which significantly increases the risk of falling in elderly people with cognitive impairment [20]. It is important to bear in mind that older people who experience even the slightest cognitive impairment usually reduce their daily activity. In the initial stage of their illness, they often give up any independent travel (walks) in unfamiliar places, for fear of getting lost. As their condition deteriorates, even the simplest activities of daily life become a major problem (e.g. an unassisted walk to a nearby shop). The family begin to relieve the patient even from the easiest self-service activities, which leads to a further reduction of the muscle strength and a deterioration of the functional ability of the patient's entire body. Therefore, a focus on maintaining the proper muscle strength, with a particular attention to the quadriceps femoris muscles, and care for the general fitness and physical capacity of the whole body should be an important element in all fall prevention programs.

As for the eyes-closed quiet stance trial, all the studied stabilographic parameters correlated significantly with the
MMSE score. Such a high correlation may be explained by a significant involvement of the visual system in the process of maintaining balance among elderly people. The results obtained by King et al. [21] suggest that among older people, the balance compensation mechanisms are based on the visual stimuli to a significantly higher degree than among young people. Similar results were obtained for people with Parkinson's disease [22].

Balance control is possible owing to the cooperation of the proprioceptive, visual, and vestibular systems [23]. Increasing the difficulty of a balance test by disturbing one of these systems results in a greater involvement of the remaining two. The above study has shown a strong link between the severity of cognitive impairment and the deterioration of stabilographic parameters. This may indicate a decreased quality of functioning of the other systems involved in the balance-keeping process among people with a lower MMSE score. On the one hand, this may result from a weaker function of the vestibular system in patients with cognitive impairment $[24,25]$. On the other hand, for the proper functioning of proprioception, it is necessary that the information fed to the central nervous system is processed efficiently, which is compromised in the case of people with cognitive impairment [26]. In consequence, in the presence of cognitive disorders, the postural stability is significantly reduced when the visual system is shut down. It seems that the key in preventing falls in older people with cognitive impairment is the improvement of the function of the two remaining systems (vestibular and proprioceptive) involved in balance control.

\section{Limitations}

The authors are aware of the limitations of the above study. Firstly, extending the duration of each trial to $90 \mathrm{sec}-$ onds could increase the sensitivity of correlation. According to literature, static stabilographic measurements prove to be the most effective when averaging three 90-second trials [27]. In the present study, one of the inclusion criteria was obtaining an appropriate MMSE score. Although this assessment tool works well for brief screening, the Montreal Cognitive Assessment (MoCA) test is believed to be much more effective in diagnosing cognitive disorders [28]. It might be worth considering to repeat our study with the inclusion criteria based on the MoCA.

\section{Conclusions}

In summary, the study indicated a significant link between the severity of cognitive impairment and the results of stabilographic parameters. A negative correlation between the stabilographic parameters and the MMSE scores showed a linear deterioration of postural stability which progressed along with a decrease in cognitive functions. This correlation turned out stronger when the difficulty of the trial was increased by excluding the input from the visual system. This result suggests that in order to counteract the increased risk of falls in the group of people with cognitive impairment, it is worth putting emphasis on the improvement of the remaining balance-maintaining systems, i.e. the vestibular and the proprioceptive system. In addition, it seems important to ensure that patients with cognitive impairment have their eyesight regularly checked and any defects corrected, as for this group vision is a very important source of information in the context of balance control. Also, in fall prevention programs, a major role should be played by exercises aiming to improve muscle strength, with a particular focus on the quadriceps femoris muscle. 


\section{Disclosure statement}

No author has any financial interest or received any financial benefit from this research.

\section{Conflict of interest}

The authors state no conflict of interest.

\section{References}

1. Wu Y-T, Prina AM, Brayne C. The association between community environment and cognitive function: a systematic review. Soc Psychiatry Psychiatr Epidemiol. 2015;50(3):351-362; doi: 10.1007/s00127-014-09456.

2. Zarzeczny R, Nawrat-Szoltysik A, Polak A, Maliszewski J, Kiełtyka A, Matyja B, et al. The correlation between selected anthropometric indices and BIA-based body fat measures in nursing home women aged $80+$ years. Phys Act Rev. 2016;4:89-99; doi: 10.16926/par.2016.04.11.

3. Barcikowska M, Bień B, Bizdan L. Diagnosis and treatment of dementias: recommendations of the Interdisciplinary Group of Experts on Diagnosing and Treating Dementias (IGERO 2006) [in Polish]. Lublin: Czelej; 2006.

4. Sachdev PS, Lipnicki DM, Kochan NA, Crawford JD, Thalamuthu A, Andrews G, et al. The prevalence of mild cognitive impairment in diverse geographical and ethnocultural regions: the COSMIC collaboration. PLoS One. 2015;10(11):e0142388; doi: 10.1371/journal.pone.0142388.

5. Winblad B, Palmer K, Kivipelto M, Jelic V, Fratiglioni L, Wahlund L-O, et al. Mild cognitive impairment - beyond controversies, towards a consensus: report of the International Working Group on Mild Cognitive Impairment. J Intern Med. 2004;256(3):240-246; doi: 10.1111/j.13652796.2004.01380.x.

6. César KG, Brucki SMD, Takada LT, Nascimento LFC, Gomes CMS, Almeida MCS, et al. Prevalence of cognitive impairment without dementia and dementia in Tremembé, Brazil. Alzheimer Dis Assoc Disord. 2016;30(3):264-271; doi: 10.1097/WAD.0000000000000122.

7. Liu-Ambrose T, Ashe MC, Graf P, Beattie BL, Khan KM. Mild cognitive impairment increases falls risk in older community-dwelling women. Phys Ther. 2008;88(12): 1482-1491; doi: 10.2522/ptj.20080117.

8. Ansai JH, de Andrade LP, Masse FAA, Gonçalves J, de Medeiros Takahashi AC, Vale FAC, et al. Risk factors for falls in older adults with mild cognitive impairment and mild Alzheimer disease. J Geriatr Phys Ther. 2017; doi: 10.1519/JPT.0000000000000135.

9. Ban B, Sevšek F, Rugelj D. A comparison of the ceiling effect between Berg Balance Scale and Mini-BESTest in a group of balance trained community-dwelling older adults. Physiother Quart. 2017;25(2):3-9; doi: 10.5114/ pq.2018.73368.

10. Cieślik B, Jaworska L, Szczepańska-Gieracha J. Postural stability in the cognitively impaired elderly: a systematic review of the literature. Dementia. 2019;18(1):178-189; doi: $10.1177 / 1471301216663012$.

11. Agrawal Y, Smith PF, Rosenberg PB. Vestibular impairment, cognitive decline and Alzheimer's disease: balancing the evidence. Aging Ment Health. 2019;1-4; doi: 10.1080/13607863.2019.1566813.

12. Bhatt J, Walton H, Stoner CR, Scior K, Charlesworth G. The nature of decision-making in people living with dementia: a systematic review. Aging Ment Health. 2018; 1-11; doi: 10.1080/13607863.2018.1544212.

13. Folstein MF, Folstein SE, McHugh PR. "Mini-mental state": a practical method for grading the cognitive state of pa- tients for the clinician. J Psychiatr Res. 1975;12(3):189198; doi: 10.1016/0022-3956(75)90026-6.

14. Tombaugh TN, Mclntyre NJ. The mini-mental state examination: a comprehensive review. J Am Geriatr Soc. 1992;40(9):922-935; doi: 10.1111/j.1532-5415.1992. tb01992.x.

15. Mesbah N, Perry M, Hill KD, Kaur M, Hale L. Postural stability in older adults with Alzheimer disease. Phys Ther. 2017;97(3):290-309; doi: 10.2522/ptj.20160115.

16. Szczepańska-Gieracha J, Cieślik B, Chamela-Bilińska D, Kuczyński M. Postural stability of elderly people with cognitive impairments. Am J Alzheimers Dis Other Demen. 2016;31(3):241-246; doi:10.1177/1533317515602547.

17. Hamed A, Bohm S, Mersmann F, Arampatzis A. Exercises of dynamic stability under unstable conditions increase muscle strength and balance ability in the elderly. Scand J Med Sci Sports. 2018;28(3):961-971; doi: 10.1111/ sms.13019.

18. Lauretani F, Maggio M, Ticinesi A, Tana C, Prati B, Gionti $L$, et al. Muscle weakness, cognitive impairment and their interaction on altered balance in elderly outpatients: results from the TRIP observational study. Clin Interv Aging. 2018;13:1437-1443; doi: 10.2147/CIA.S165085.

19. Moxley Scarborough D, Krebs DE, Harris BA. Quadriceps muscle strength and dynamic stability in elderly persons. Gait Posture. 1999;10(1):10-20; doi: 10.1016/S09666362(99)00018-1.

20. Cieślik B, Ostrowska B. The relation between cognitive impairment and the risk of falling among elderly people: preliminary reports. Sport i Turystyka Środkowoeuropejskie Czasopismo Naukowe. 2018;1(2):117-125. doi: 10.16926/sit.2018.01.18

21. King GW, Abreu EL, Kelly PJ, Brotto M. Neural control of postural sway: relationship to strength measures in young and elderly adults. Exp Gerontol. 2019;118:3944; doi: 10.1016/j.exger.2019.01.005.

22. Da Silva TC, Felippe LA, Carregaro RL, Christofoletti G. Postural instability in subjects with Parkinson's disease undergoing different sensory pitfalls. Hum Mov. 2017; 18(4):55-60; doi: 10.1515/humo-2017-0031.

23. Fitzpatrick R, McCloskey DI. Proprioceptive, visual and vestibular thresholds for the perception of sway during standing in humans. J Physiol. 1994;478(Pt 1):173-186; doi: 10.1113/jphysiol.1994.sp020240.

24. Nakamagoe K, Kadono K, Koganezawa T, Takiguchi M, Terada M, Yamamoto F, et al. Vestibular impairment in frontotemporal dementia syndrome. Dement Geriatr Cogn Dis Extra. 2016;6(2):194-204; doi: 10.1159/000445870.

25. Smith PF. The vestibular system and cognition. Curr Opin Neurol. 2017;30(1):84-89; doi: 10.1097/WCO.00 00000000000403.

26. Adamo DE, Martin BJ, Brown SH. Age-related differences in upper limb proprioceptive acuity. Percept Mot Skills. 2007;104(3 Pt 2):1297-1309; doi: 10.2466/pms.104.4. 1297-1309.

27. Alsalaheen B, Haines J, Yorke A, Broglio SP. Reliability and construct validity of limits of stability test in adolescents using a portable forceplate system. Arch Phys Med Rehabil. 2015;96(12):2194-2200; doi: 10.1016/j. apmr.2015.08.418.

28. Ciesielska N, Sokołowski R, Mazur E, Podhorecka M, Polak-Szabela A, Kędziora-Kornatowska K. Is the Montreal Cognitive Assessment (MoCA) test better suited than the Mini-Mental State Examination (MMSE) in mild cognitive impairment (MCl) detection among people aged over 60? Meta-analysis. Psychiatr Pol. 2016;50(5): 1039-1052; doi: 10.12740/PP/45368. 